$\Rightarrow$ POROUS MATERIALS

\title{
A new class of glass
}

\section{6 \\ there is more to obtaining porous glasses than rapidly cooling any liquid MOF}

Many technologies - from medical devices to sensors - rely on porous glasses to serve as robust and selective hosts for small molecules. Microporous glasses are particularly valuable and can be made from modular metal-organic frameworks (MOFs). A recent study describes a new method for making porous MOF-derived glasses with pores that are reversibly accessible to guest molecules such as $\mathrm{CO}_{2}$. The method relies on the rapid cooling of a liquid MOF, such that no chemical modification is needed to introduce porosity or function. As disclosed in Nature Communications, this potentially general route to microporous glasses is the culmination of a collaborative effort led by Thomas Bennett and Shane Telfer.
"Tom being interested in glasses and myself being keen on porosity, it was a natural evolution to look for porous glasses," says Telfer.

Bennett's group had previously shown that melting zeolitic imidazolate frameworks (ZIFs, members of a class of MOF) affords porous liquids that lack the crystallinity of solid ZIFs but retain the molecular-level connectivity and porosity. Melt-quenching the liquid ZIF by rapidly cooling it produces a glass. "We have recently shown that the mechanical properties of these MOF glasses are interesting when compared to other glasses," says Bennett. "However, to compare favourably with crystalline MOFs, porosity needed to be introduced." Porous glasses are known and include examples based on MOFs with accessible pores. But melt-quenching a ZIF such as [Zn(imidazolato) (5-chlorobenzimidazolato $)_{2-n}$ ] is especially interesting because when hot, the material can be manipulated into useful shapes, such as thin films.

Once cooled, the materials are intrinsically porous, which is not the case with traditional porous glasses that require subsequent processing. Furthermore, the glassy MOF retains a glass-to-liquid transition and can thus, in principle, be melted and reshaped before requenching.

It is important to note that there is more to obtaining porous glasses than rapidly cooling any liquid MOF. Bennett's team had earlier melt-quenched

ZIFs to obtain glasses featuring a network of pores inaccessible to small molecules. Opening up this network to guest molecules - key to many proposed applications of MOFs - required some clever molecular design. "We prepared MOFs with one small organic linker, to facilitate melting, and one larger one, to stop the whole system from 'caving in' when it does melt," explains Bennett. "These act a bit like struts to create pockets of porosity when everything is falling in around them."

Realizing this design brought together experts from 11 institutions in 6 countries, including solidstate NMR specialists in Slovenia, beamline scientists from Oxfordshire, UK and a computational chemist in Turkey. "The work really leads itself when you have such great people involved," says Bennett.

Relative to crystalline MOFs, the new glasses have a lower pore volume and a correspondingly lower capacity for guest molecules. However, the authors argue that simply comparing the glasses with high-capacity crystalline MOFs overlooks advantages of meltquenching. For example, their bottom-up approach should enable improvements to be made by altering the structure of the parent MOF. This will require an understanding of the structural requirements that enable a MOF to be converted into a porous glass. "We are starting to learn the rules that govern whether a ZIF will produce a glass or not," says Telfer. "From here, we hope that the design rules for producing porous glasses can be fully elucidated." This promises a degree of versatility, and both Bennett and Telfer expect to see more MOF glasses with accessible porosity — including those with higher capacities - to be uncovered soon.

Andrew Bissette

ORIGINAL ARTICLE Zhou, C. et al. Metal-organic framework glasses with permanent accessible porosity. Nat. Commun. 9, 5042 (2018) FURTHER READING Gaillac, R. et al. Liquid metal-organic frameworks. Nat. Mater. 16, 1149-1154 (2017) 